\title{
Insuficiencia hepática crónica y hemostasia
}

\section{Chronic Liver Failure and Hemostasis}

Christian Ramos P., ${ }^{1}$ Adrián Santoyo S., ${ }^{1}$ Humberto Castellanos S., ${ }^{1}$ Carlos Martínez M., ${ }^{1}$ Irma Olarte C., ${ }^{2}$ Adolfo Martínez M. ${ }^{2}$

1 Servicio de hematología, Hospital General de México, Ciudad de México, México.

2 Laboratorio de biología molecular, Hospital General de México, Ciudad de México, México. Correspondencia: Christian Omar Ramos-Peñafiel, MD. Correo electrónico: leukemiachop@hotmail.com.

Fecha recibido: $31-10-16$

Fecha aceptado: 06-10-17

\begin{abstract}
Resumen
La insuficiencia hepática es un estado patológico que condiciona la síntesis y metabolismo de diversas biomoléculas, siendo las alteraciones a la hemostasia una de las primeras consecuencias a nivel sistémico que se hacen presentes; debido a esto y por la dimensión de los riesgos de esta situación, no es raro que las pruebas de coagulación sean indispensables para formular las escalas pronósticas en pacientes hepatópatas.

Los conocimientos sobre hemostasia han avanzado en la última década; la clásica cascada de activación de los factores de la coagulación ha sido perfeccionada hasta conformarse el ahora vigente modelo celular que considera la valiosa e indispensable participación del endotelio y las plaquetas. Gracias a esto es posible comprender que, en pacientes con insuficiencia hepática, el riesgo de sangrado no obedece únicamente a la deficiencia en la producción de los factores de la coagulación y, por tanto, es cuestionable la administración de vitamina K. Más relevante aún es que, gracias a estos conocimientos, se puede comprender el a veces contradictorio riesgo de trombosis en estos pacientes, complicación potencialmente mortal.
\end{abstract}

\section{Palabras clave}

Coagulación sanguínea, insuficiencia hepática, trombosis, vitamina K.

\section{Abstract}

Hepatic insufficiency is a pathology that conditions synthesis and metabolism of various biomolecules. Alterations of hemostasis is one of its first systemic consequences. Because of this and the size of the risks, it is not uncommon for clotting tests to be indispensable for formulating prognostic scales in patients with liver disease.

Knowledge about hemostasis has advanced in the last decade, and the classic cascade of activation of coagulation factors has been perfected until it has become the now-current cellular model that considers the valuable and indispensable participation of the endothelium and platelets. Thanks to this, it is possible to understand that the risk of bleeding in patients who have hepatic insufficiency is not only due to deficiencies in production of coagulation factors, and that for this reason administration of vitamin $\mathrm{K}$ is questionable. Even more relevant, is the fact that, thanks to this this knowledge, we can understand the sometimes contradictory and potentially life-threatening complication of thrombosis in these patients.

\section{Keywords}

Blood coagulation, liver failure, thrombosis, vitamin $\mathrm{K}$. 


\section{INTRODUCCIÓN}

El hígado es un órgano vital que realiza diversos procesos metabólicos que van desde la transformación y eliminación de toxinas, hasta el almacenamiento y producción de biomoléculas indispensables para la vida. Se denomina insuficiencia o falla hepática cuando el hígado está incapacitado para llevar a cabo sus funciones de síntesis y metabolismo; inicialmente se puede clasificar la falla hepática como aguda o crónica, y recientemente se reconoce un nuevo estado caracterizado por una exacerbación aguda sobre un daño hepático crónico $(1,2)$.

La lesión inducida por fármacos es la principal causa de falla hepática, que se subdivide de acuerdo con el tipo de daño ocasionado: intrínseca (dependiente de la dosis), cuyo mejor ejemplo es el acetaminofén; e idiosincrática (independiente de la dosis) (3-5). Las causas más frecuentes de falla hepática crónica son la enfermedad hepática por alcohol, seguida de la ocasionada por agentes infecciosos (75\%-80\% asociada con el virus de la hepatitis C [VHC]). Algunos expertos consideran que los estados proinflamatorios asociados con esteatosis, como la obesidad o la diabetes, también deben ser considerados como factores para el desarrollo de falla hepática crónica. Las enfermedades autoinmunes merecen mención particular porque pueden manifestarse con una insuficiencia hepática aguda, o causar un deterioro crónico (Tabla 1) $(6,7)$.

Tabla 1. Clasificación y agentes causantes de insuficiencia hepática

\begin{tabular}{lll}
\hline \multicolumn{1}{c}{ Aguda } & \multicolumn{1}{c}{ Crónica agudizada } & \multicolumn{1}{c}{ Crónica } \\
\hline Alcohol & Alcohol & Alcohol \\
Autoinmunidad & Autoinmunidad & Autoinmunidad \\
Fármacos: & & Infecciones: \\
AINE & Hepatitis B \\
Estatinas & Hepatitis C \\
Suplementos & Estados \\
alimenticios & proinflamatorios: \\
Quimioterapia & Obesidad \\
Antibióticos & Diabetes \\
Antifúngicos & \\
Antituberculosos & \\
(isoniazida) & \\
\hline
\end{tabular}

AINE: antiinflamatorios no esteroideos.

\section{FISIOLOGÍA DE LA HEMOSTASIA}

La hemostasia es un proceso complejo que involucra la estimulación de factores de coagulación sobre una superficie de plaquetas activadas para lograr la reparación del tejido vascular (8). En general, la hemostasia se divide en primaria, mediada por el endotelio y las plaquetas con el fin de integrar el tapón hemostático; y secundaria, que implica el proceso de coagulación $(8,9)$.

Durante las últimas décadas se ha logrado tener una mejor comprensión de la hemostasia. Uno de los cambios más relevantes es dejar de considerar a las plaquetas únicamente como los elementos formes que integran el tapón plaquetario durante la hemostasia primaria, ahora se sabe que poseen más de 300 sustancias contenidas en los gránulos intracelulares (gránulos $\alpha$ ), entre los que se destacan los factores de crecimiento necesarios para la regeneración tisular (10).

\section{Modelo celular de la coagulación}

Tradicionalmente, la coagulación era entendida como una activación ordenada de factores en forma de cascada, pero actualmente el modelo propuesto por Hoffman y Monroe permite una mejor comprensión al considerar las interacciones entre los factores y los elementos celulares (11). El modelo celular de la coagulación parte del estado basal donde el factor X y VII se encuentran inactivos en el torrente sanguíneo y existen células portadoras (endotelio, subendotelio y monocitos) del factor tisular (FT, o también conocido como factor III), ante los estímulos nocivos el FT es liberado al torrente sanguíneo y comienza propiamente el proceso de la coagulación que consta de 3 fases:

1. Fase 1 o iniciación: el FT se une al factor VII para transformar y activar los factores IX y X. El factor Xa se combina con el factor Va presente en las superficies celulares, transformando pequeñas cantidades de protrombina (factor II) a trombina (factor IIa).

2. Fase 2 o amplificación: la trombina mantiene la producción del factor Xa mediante su intervención en la vía intrínseca (factores XI y IX), y de forma más relevante al liberar de su transportador plasmático y activar el factor VIII, siendo el factor VIIIa un catalizador altamente eficiente del factor X. En conjunto, las vías mencionadas hasta el momento tienen como finalidad mantener e incrementar sustancialmente la producción de trombina, siendo un ambiente propicio para reclutar y activar más plaquetas.

3. Fase 3 o propagación: las plaquetas, una vez activadas, liberan sustancias quimioatrayentes como el difosfato de adenosina $(\mathrm{ADP})$ y el tromboxano $\mathrm{A}_{2}$, además de cambiar su conformación física a una conformación que facilita la creación del tapón hemostático y expresar en su superficie fosfolípidos de carga negativa como la fosfatidilserina, los cuales en conjunto con el calcio sirven como templete para la formación de los complejos tenasa (factor VIIIa y IXa) y protrombinasa (factor $\mathrm{Xa}$ y Va), que tienen la capacidad de generar grandes cantidades de trombina. Finalmente, la trombina con- 
diciona la polimerización de fragmentos de fibrinógeno en fibras de fibrina que darán estabilidad al coágulo inicial. Adicionalmente, la trombina activa al factor XIII, que estabilizará a la fibrina y brindará mayor resistencia al proceso de lisis (Figura 1) $(9,12,13)$.

\section{FISIOPATOLOGÍA DE LA HEMOSTASIA DURANTE LA INSUFICIENCIA HEPÁTICA}

\section{Alteraciones en la hemostasia primaria}

Desde etapas tempranas, en la enfermedad hepática existe una acumulación masiva de lípidos en el hepatocito que inducen la liberación de diversos mediadores inflamatorios, entre los que se pueden mencionar la interleucina (IL) 1 y 6, el factor de necrosis tumoral (TNF) y la estimulación del receptor LPS/Toll-like (TLR-4) presentes en las células de $\operatorname{Kupffer}(14,15)$. Estas vías desencadenan una disregulación del endotelio mediante la activación de la dimetilarginina asimétrica (ADMA), un inhibidor endógeno de la óxido nítrico sintetasa (eNOS) $(16,17)$. Gracias a modelos animales, ahora se sabe que la disminución de la actividad de la eNOS es parte fundamental de la fisiopatología de la hipertensión portal $(18,19)$.

Las plaquetas, además de servir como elemento celular en el tapón hemostático, poseen gránulos intracelulares con elementos capaces de modificar los procesos inflamatorios e inmunológicos dentro del hígado (10). Los gránulos a contienen factores de crecimiento cuya diana son las vías Akt (fosfatidilinositol-3-kinasa/AKT [PI3K-Akt]) y extracellular signal-regulated kinase (ERK) 1/2, ambas trascendentales para el hepatocito (20) al grado que recientes investigaciones sugieren que la transfusión de plaquetas puede mejorar la regeneración hepática $(20,21)$. Los gránulos densos contienen nucleótidos de adenina que inactivan a las células estelares del espacio intersinusoidal y, en consecuencia, frenan los mecanismos profibróticos $(21,22)$.

La trombocitopenia es una de las manifestaciones más frecuentes de la enfermedad hepática. Alrededor de un 76\%-85\% de los pacientes desarrolla algún grado de trombocitopenia $\left(<150 \times 10^{3} / \mu \mathrm{L}\right)$, aunque solo un $13 \%$ presenta cifras $<50 \times 10^{3} / / \mu \mathrm{L}(23)$. Cabe recordar que el principal

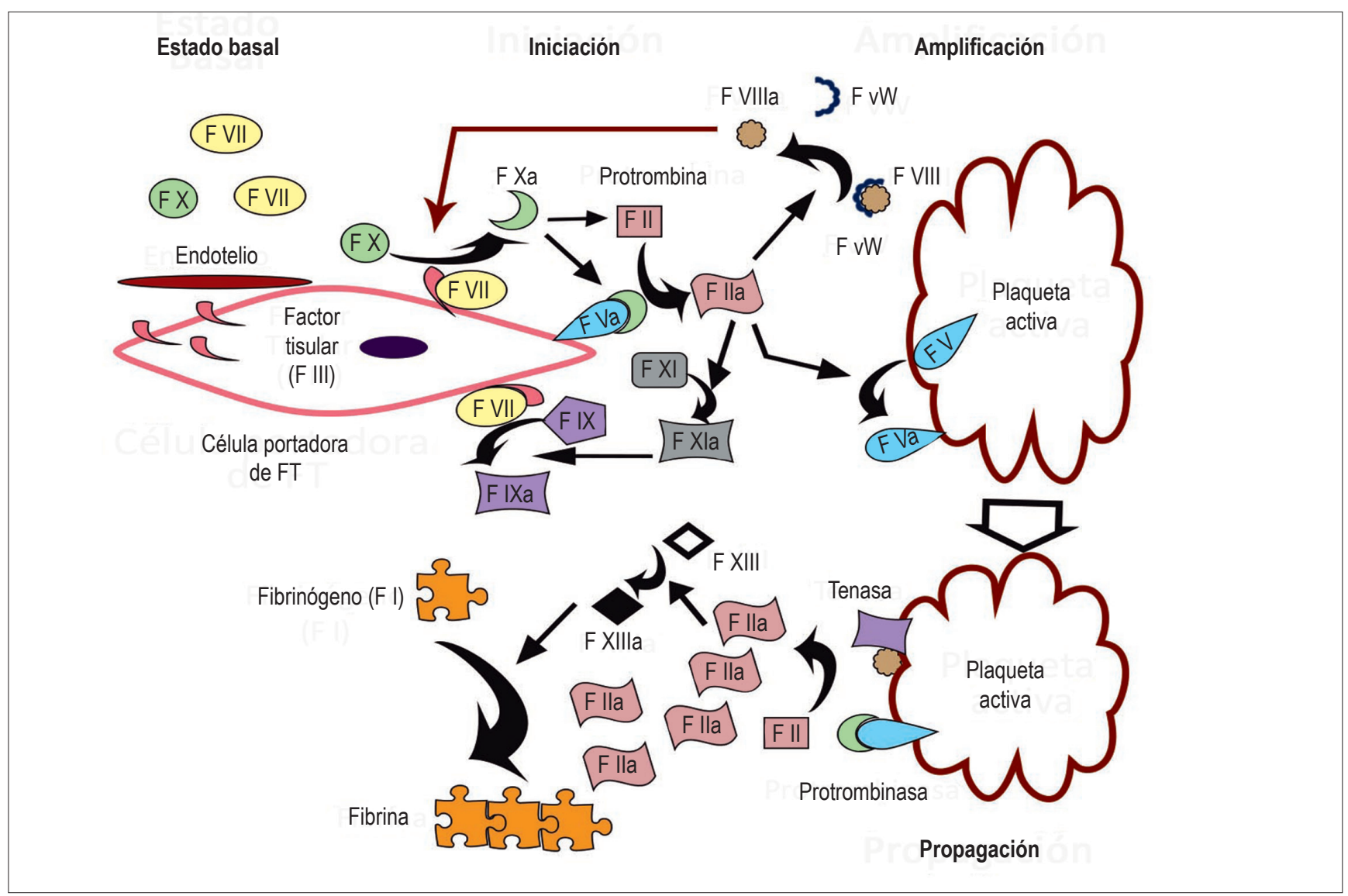

Figura 1. Modelo celular de la coagulación. F: factor. 
estímulo para la formación de plaquetas es la trombopoyetina (TPO), hormona producida primordialmente por los hepatocitos (24). La unión de la TPO con su receptor c-Mpl promueve las cascadas de activación de la quinasa de Janus 2 (JAK2) y la tirosina quinasa 2 (TYK2), además de hacer sinergia con otros estimulantes como la IL-3, IL-11 y el factor de célula progenitora (stem-cell factor), resultando en la proliferación y maduración de los megacariocitos $(24,25)$. Panasiuk y colaboradores demostraron que en pacientes con cirrosis hepática existía una disminución de la producción de TPO y, en consecuencia, una disminución de plaquetas, así como una asociación directa entre la cuenta plaquetaria y el factor de crecimiento derivado de los hepatocitos $(\mathrm{p}<0,01)(26)$. Bajo este contexto se iniciaron ensayos clínicos sobre el uso de miméticos de la TPO en casos de falla hepática, especialmente en pacientes con hepatitis crónica viral. Al respecto, $\mathrm{McHutchison} \mathrm{y}$ colaboradores evaluaron la adición del agonista del receptor de TPO (eltrombopag) a diferentes dosis $(30,50$ y 75 $\mathrm{mg}$ ) contra placebo durante 4 semanas en portadores de VHC que no podían iniciar terapia antiviral por cursar con trombocitopenia severa. La mejor respuesta se observó en el grupo de eltrombopag a $75 \mathrm{mg}$, en el que el $95 \%$ de pacientes elevó significativamente su cifra de plaquetas a niveles aceptables (27); Afdhal y colaboradores obtuvieron resultados favorables similares en las cohortes endothelin antagonist bosentan for lowering cardiac events (ENABLE) 1 y 2 con 9 semanas de seguimiento (28). Este mismo autor reportó en el estudio early levosimendan vs usual care in advanced chronic heart failure (ELEVATE) los beneficios de emplear eltrombopag para la elevación de la cifra de plaquetas antes de la realización de procedimientos invasivos (75 mg durante 14 días antes del procedimiento), lo que disminuye los requerimientos transfusionales en un $72 \%$, aunque con un incremento de la trombosis de la vena porta en comparación con placebo (29).

Dentro de la fisiopatología de la trombocitopenia también se aprecia un incremento en la destrucción de plaquetas debido a hiperesplenismo o mediado por anticuerpos. Para diferenciar entre ambos mecanismos, en el primero se aprecia una trombocitopenia moderada acompañada también de una disminución de leucocitos por secuestro esplénico $(30,31)$. Al respecto existen 2 grandes series que señalan a la esplenectomía (abordaje tradicional o laparoscópico) como medida terapéutica que ayuda a elevar las cifras de plaquetas $\left(>100 \times 10^{3} / \mu \mathrm{L}\right)$ y leucocitos en pacientes con cirrosis hepática $(32,33)$. En modelos animales de fibrosis hepática se ha demostrado que la esplectomía reduce los niveles de factor de crecimiento transformante $\beta$ (TGF- $\beta$ ), por esto se logra limitar e incluso reducir las áreas de cirrosis, además de cambios en el perfil inmunológico (incremento de linfocitos CD8+) $(34,35)$.

\section{Alteraciones en la hemostasia secundaria}

La mayor parte de los factores procoagulantes como el fibrinógeno, la protrombina y los factores V, VII, IX, X, XI y XII son de síntesis hepática, por lo que sus niveles séricos se modifican en relación con el grado de daño hepático (36, 37). Los factores V y VIII son los primeros en disminuir por poseer las vidas medias más cortas ( 12 y 4-6 horas, respectivamente) (38). Es común que los pacientes cirróticos sufran reducciones moderadas del factor VII, identificándose una relación significativa entre los niveles de factor VII y el tiempo de protrombina (TP) $(\mathrm{r}=0,76, \mathrm{p}<0,001)$ (39).

Cuando se evalúan las pruebas de coagulación en pacientes con hepatopatías, es importante descartar defectos congénitos; el más frecuente es la deficiencia de factor VII ( 1 caso por cada 500000 nacimientos) (40). Esta deficiencia congénita se ha descrito en varias entidades clínicas (embarazo, cardiopatía, enfermedad pulmonar obstructiva, entre otras), aunque se tienen registrados pocos casos en pacientes cirróticos (41).

\section{TROMBOSIS E INSUFICIENCIA HEPÁTICA}

Previamente se mencionaron en detalle las vías moleculares de la trombina, y se mencionó su capacidad para generar estados de microtrombosis y consecuentes estados de isquemia en los sinusoides, lo que resulta en la expresión de factores profibrogénicos, que a su vez acelera y perpetúa el daño al parénquima hepático (42). Con este contexto no es raro que los pacientes con insuficiencia hepática, a pesar de ser proclives a eventos hemorrágicos, sufran de un estado de trombofilia altamente localizado en el sistema venoso esplácnico, cuya traducción clínica es alguna de las siguientes entidades: síndrome de Budd-Chiari (SBC), trombosis mesentérica ( 1 caso por millón de personas) y trombosis extrahepática de la vena porta (4 casos por millón de personas) (43); cabe recordar que hasta el $40 \%$ de los pacientes con cirrosis hepática sufre de trombosis de la vena porta $(19,44-46)$. Algunos expertos sugieren que la reducción de velocidad de flujo en la vena porta es un hallazgo predictor de eventos trombóticos (45), por lo que una vez detectado debe iniciarse alguna estrategia antitrombótica, entre las que se pueden citar: terapia anticoagulante, angioplastia o colocación de endoprótesis vascular (stent), realización de una derivación transyugular intrahepática o incluso considerar trasplante hepático $(47,48)$.

\section{Factor de von Willebrand (FvW)}

El FvW es una glucoproteína almacenada en los cuerpos de Weibel-Palade al interior de las células endoteliales y, una vez secretado, el FvW favorece la adhesión plaquetaria, ade- 
más de unirse al factor VIII para evitar su pronta degradación (49). Durante la progresión del daño hepático existe una disminución de factores de coagulación, pero contrario a lo pensado también existe una elevación paralela del FvW y del factor VIII (50); el mecanismo subyacente parece ser la mayor actividad endotelial, el lugar de síntesis del FvW y el sitio donde se ubican las células involucradas en los procesos de fibrosis hepática que desembocarán en la reducción de los niveles de proteína C, S (factores anticoagulantes) y de la metaloproteasa ADAMTS $_{13}$ (a disintegrin and metalloproteinasa with thrombospondin type 1 motif $n .{ }^{\circ} 13$ ) (44). Esta última es una proteasa sintetizada por las células estelares y es la encargada de fraccionar el FvW en pequeños multímeros capaces de unirse al factor VIII $(51,52)$; la disminución de su actividad $(<10 \%)$ causada por mutaciones, autoanticuerpos o por una producción reducida (insuficiencia hepática) da como resultado la producción de multímeros de FvW ultralargos (UL-FvW) que no pueden unirse al factor VIII, esto resulta en el incremento de factor VIII en su forma libre y activa $(52,53)$. Un grupo de investigadores de la universidad de Nara, en Japón, han evidenciado que, en pacientes con insuficiencia hepática por alcohol, la actividad de ADAMTS $_{13}$ disminuye y los niveles séricos de UL-FvW se incrementan; pero más importante aún es que estos hechos se correlacionan con el grado de severidad del daño hepático, e incluso plantean la posibilidad de emplearse como marcadores pronósticos $(53,54)$.

\section{Proteína C y S}

La proteína $\mathrm{C}$ es uno de los principales anticoagulantes producidos en el hígado como un cimógeno que se activa en presencia del complejo trombina-trombomodulina sobre las superficies endoteliales. Una vez activada (proteína C activa, PCA) inhibe a 2 de los principales cofactores de la coagulación: factor Va y VIIIa (55).

La proteína S, codificada por el gen PROS1 y producida por los hepatocitos, células endoteliales, megacariocitos y osteoblastos, es parte fundamental no solo de la coagulación sino también de la ateroesclerosis, angiogénesis y la progresión de células neoplásicas (56). Es dependiente de la vitamina $\mathrm{K}$ y actúa estimulando la producción de PCA para regular la coagulación (57).

Durante el estudio de pacientes con eventos trombóticos debe tenerse en cuenta la disminución y falta de activación de estas proteínas debido al daño hepático, pero también descartarse otras situaciones clínicas concomitantes como resistencia a la PCA, defectos hereditarios de la coagulación (deficiencia de proteína C, S, factor V, Leiden, Factor II, mutaciones en metilentetrahidrofolato [MTHF] reductasa o hiperhomocistinemia) síndrome de antifosfolípidos e inclusive neoplasias $(57,58)$; esto último en relación con el hecho de que existen homólogos de la proteína $S$ en algunos tumores hepáticos $(59,60)$.

\section{VALORACIÓN DE LA HEMOSTASIA EN PACIENTES HEPATÓPATAS}

La hemostasia es una balanza que equilibra el gran peso de los factores procoagulantes y anticoagulantes; ante la deficiencia o incremento de alguno de estos, la balanza se moverá únicamente a favor del lado dominante. En pacientes con enfermedad hepática, esta balanza tendrá un precario equilibrio por la disminución de factores en ambos lados, el más mínimo aumento o disminución de alguno de los elementos ocasionará movimientos erráticos de la aguja de la balanza hasta que encuentre y marque el sitio dominante (Figura 2) $(23,36,61)$. Se debe tener en cuenta esta comparación al momento de comenzar la valoración del estado hemostático en pacientes con enfermedad hepática, que será realizada al inicio con pruebas rutinarias (como la biometría hemática) y pruebas de coagulación básicas (TT: tiempo de trombina; TP: tiempo de protrombina; INR: international normalized ratio; ТTРa: tiempo de tromboplastina parcial activado). Estas pruebas permiten evaluar únicamente los factores procoagulantes $(23,36)$ y, salvo que se tenga una fuerte sospecha de algún defecto congénito, se puede complementar la evaluación con determinaciones de los factores de la coagulación $(40,61)$.

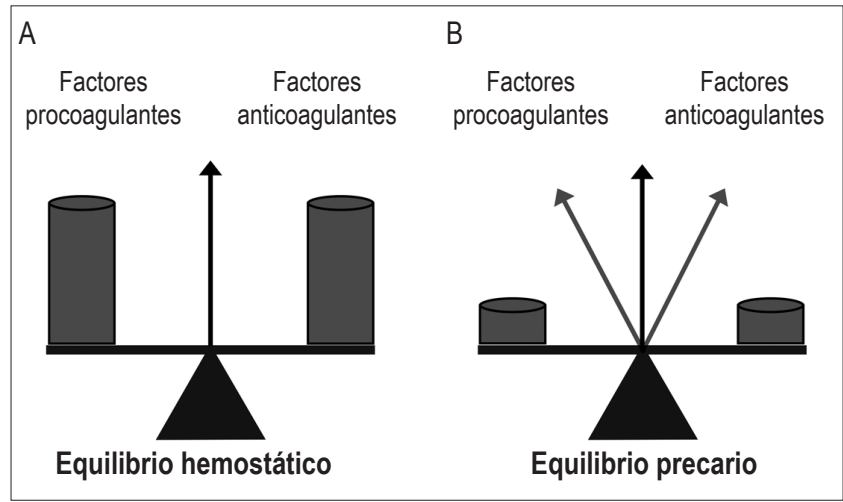

Figura 2. Equilibrio hemostático en personas sanas (A) y en pacientes con enfermedad hepática (B).

Es importante decir que el TP y TTPa son pobres predictores de sangrado en personas hepatópatas; es decir, una cifra dentro del rango habitual no garantiza protección contra sangrado, ni cifras elevadas son sinónimo de sangrados incontrolables que ameriten medidas terapéuticas correctivas o preventivas $(61,62)$. Aún se continúa en la búsqueda del método que permita determinar con fiabilidad el estado hemostático real de los pacientes con falla hepática, algunos de los más prometedores hasta el 
momento son el ratio de la generación de trombina y la tromboelastografía, aunque estas distan mucho aún de volverse pruebas rutinarias $(61,62)$.

\section{VITAMINA K}

La vitamina $\mathrm{K}$ (de la palabra alemana koagulationsvitamin, vitamina coagulante) está compuesta por un grupo de moléculas liposolubles derivadas de la 2-metil-1,4-naftoquinona (63); la filoquinona, de origen vegetal, se conoce como vitamina $K_{1}$; la menaquinona, de origen bacteriano, se denomina vitamina $K_{2}$; y las formas solubles en agua son la menadiona (vitamina $\mathrm{K}_{3}$ ) y el menadiol (vitamina $\left.\mathrm{K}_{4}\right)(63,64)$.

La vitamina $\mathrm{K}$, al ser ingerida por vía oral, se absorbe en el intestino, pasa a la linfa y, ulteriormente, al torrente sanguíneo, donde se transporta del $75 \%$ al $90 \%$ hasta el hígado por diversas lipoproteínas de alta densidad, principalmente la apolipoproteína E (ApoE) (Figura 3). Este complejo no solo sirve de transporte, también facilita la unión e internalización desde el espacio de Disse hasta el interior del hepatocito (65). La captación rápida de filoquinona en el hígado se evidencia mediante la excreción de sus metabolitos en las sales biliares (30\%-40\%); otras vías de excreción son la orina $(15 \%)$ y heces $(65,66)$.

En hemostasia, la vitamina K cataliza la carboxilación de los factores de coagulación de síntesis hepática (VII, IX, $\mathrm{X}$ y protrombina) formando sitios de unión con el calcio en los residuos de ácido glutámico a los lados de la cadena proteica. Las proteínas $\mathrm{C}$ y $\mathrm{S}$, anticoagulantes naturales, también requieren de vitamina $\mathrm{K}$ para su actividad (66,
67). Debido a esto, es fácil comprender que los pacientes con hepatopatías crónicas importantes no responden adecuadamente a la suplementación con vitamina $\mathrm{K}$ y las alteraciones de la coagulación se vuelven de difícil control.

\section{Administración de vitamina K}

La principal indicación para la administración de vitamina $\mathrm{K}$ es la deficiencia de la misma en el recién nacido, alteración poco frecuente $(0,1 \%$ de todos los recién nacidos vivos), pero potencialmente letal (65) y puede prevenirse con la administración de $2 \mathrm{mg}$ vía oral en todos los recién nacidos $(68,69)$. La segunda indicación terapéutica es como antídoto en pacientes bajo un régimen de anticoagulación con antagonistas de vitamina $\mathrm{K}$ y que sufren de hemorragia activa o alto riesgo de sangrado. Usualmente, se emplea vitamina $\mathrm{K}_{1}$ a una dosis media de $10-20 \mathrm{mg}$ intravenosa (IV) o intramuscular (IM) (administración lenta, en un lapso no menor de 30 segundos) en conjunto con plasma fresco congelado o concentrados protrombínicos $(70,71)$, medida que habitualmente en un lapso no mayor de 2 horas permite reducir el INR a niveles habituales; en casos severos, una segunda dosis similar puede administrarse en 8-12 horas $(66,72)$.

Prescribir vitamina $\mathrm{K}$ para revertir las alteraciones en la hemostasia de los pacientes con daño hepático es una práctica constante por diversos especialistas bajo la hipótesis de que existe una deficiencia de factores de la coagulación dependientes de la vitamina $\mathrm{K}$ (73); sin embargo, es importante puntualizar que la mayor parte de los episodios hemo-

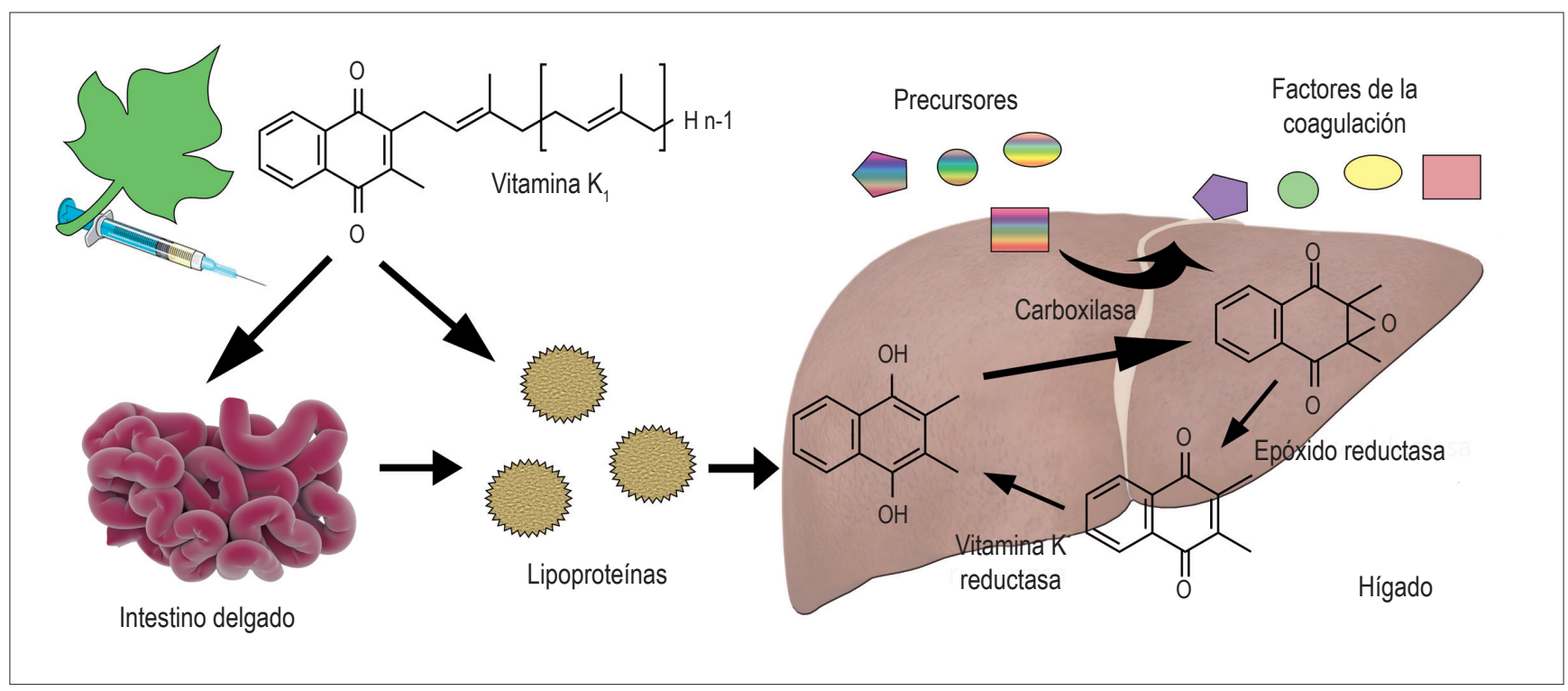

Figura 3. Metabolismo de la vitamina K. 
rrágicos no los ocasiona una deficiencia en la hemostasia secundaria, sino más bien la ruptura de várices esofágicas o úlceras gástricas secundarias a hipertensión portal por el estado de trombofilia localizado (74). De acuerdo con la revisión sistemática de Martí-Carvajal y colaboradores, no hay ningún beneficio de la administración de vitamina K en pacientes con insuficiencia hepática y hemorragia, aunque esta afirmación se basa en series de casos o estudios observacionales, faltando aún el sustento de ensayos clínicos aleatorizados (75).

\section{CONSIDERACIONES FINALES}

Conocer las últimas evidencias científicas sobre el proceso de sangrado-coagulación permite comprender mejor por qué los pacientes con falla hepática pueden desarrollar alteraciones hemostáticas desde estadios tempranos de la enfermedad hepática, razón por la que es indispensable valorar la hemostasia secundaria mediante el TP o el INR, datos que además son necesarios para aplicar las escalas pronósticas habituales en hepatópatas.

Sin embargo, ahora se sabe que el principal factor de riesgo asociado con la persistencia de hemorragia es la fibrinólisis; así que, ante un evento hemorrágico, se debe poner igual o mayor importancia al hecho de brindar un soporte transfusional adecuado, acción que ha demostrado impactar positivamente en el pronóstico a corto plazo. Así entonces, el soporte transfusional puede indicarse solo de manera profiláctica (pacientes sin hemorragia que serán sometidos a biopsia hepática u otros procedimientos invasores) o con fines terapéuticos (hemorragia digestiva); en este último caso, con mayor sustento fisiopatológico que la administración empírica de vitamina $\mathrm{K}$. Antes de iniciar el soporte transfusional, es importante considerar las complicaciones inherentes a la transfusión (formación de aloanticuerpos) y alteraciones hemodinámicas propias de pacientes con falla hepática como la lesión pulmonar aguda asociada con la transfusión (TRALI) o la sobrecarga de volumen (es importante recordar que la transfusión de plasma y plaquetas son las principales causas de TRALI) $(76,77)$. También se hizo mención de la aparente utilidad de fármacos miméticos de la TPO para abatir trombocitopenias y evitar el uso de soporte transfusional, aunque la evidencia aún es limitada, por lo que en este momento aún no puede recomendarse su uso generalizado.

\section{REFERENCIAS}

1. Bernal W, Jalan R, Quaglia A, et al. Acute-on-chronic liver failure. Lancet. 2015;386(10003):1576-87. https://doi. org/10.1016/S0140-6736(15)00309-8
2. Wang Q, Yang F, Miao Q et al. The clinical phenotypes of autoimmune hepatitis: A comprehensive review. J Autoimmun. 2016;66:98-107. https://doi.org/10.1016/j. jaut.2015.10.006

3. Licata A. Adverse drug reactions and organ damage: The liver. Eur J Intern Med. 2016;28:9-16. https://doi. org/10.1016/j.ejim.2015.12.017

4. Leise MD, Poterucha JJ, Talwalkar JA. Drug-induced liver injury. Mayo Clin Proc. 2014;89(1):95-106. https://doi. org/10.1016/j.mayocp.2013.09.016

5. Rangnekar AS, Fontana RJ. An update on drug induced liver injury. Minerva Gastroenterol Dietol. 2011;57(2):213-29.

6. Dugum M, McCullough A. Diagnosis and management of alcoholic liver disease. J Clin Transl Hepatol. 2015;3(2):10916. https://doi.org/10.14218/JCTH.2015.00008

7. Modaresi Esfeh J, Ansari-Gilani K. Steatosis and hepatitis C. Gastroenterol Rep (Oxf). 2016;4(1):24-9.

8. Versteeg HH, Heemskerk JW, Levi M, et al. New fundamentals in hemostasis. Physiol Rev. 2013;93(1):327-58. https://doi.org/10.1152/physrev.00016.2011

9. Stassen JM, Arnout J, Deckmyn H. The hemostatic system. Curr Med Chem. 2004;11(17):2245-60. https://doi. org/10.2174/0929867043364603

10. Golebiewska EM, Poole AW. Platelet secretion: from haemostasis to wound healing and beyond. Blood Rev. 2015;29(3):153-62. https://doi.org/10.1016/j. blre.2014.10.003

11. Roberts HR, Hoffman M, Monroe DM. A cell-based model of thrombin generation. Semin Thromb Hemost. 2006;32 Suppl 1:32-8. https://doi.org/10.1055/s-2006-939552

12. Nilsson IM. Coagulation and fibrinolysis. Scand J Gastroenterol Suppl. 1987;137:11-8. https://doi. org/10.3109/00365528709089754

13. Chan AK, Paredes N. The coagulation system in humans. Methods Mol Biol. 2013;992:3-12. https://doi. org/10.1007/978-1-62703-339-8_1

14. Tilg H, Moschen AR, Szabo G. Interleukin-1 and inflammasomes in alcoholic liver disease/acute alcoholic hepatitis and nonalcoholic fatty liver disease/nonalcoholic steatohepatitis. Hepatology. 2016;64(3):955-65. https://doi. org/10.1002/hep. 28456

15. Kawaratani H, Tsujimoto T, Douhara A, et al. The effect of inflammatory cytokines in alcoholic liver disease. Mediators Inflamm. 2013;2013:495156. https://doi. org/10.1155/2013/495156

16. Yang YY, Lee TY, Huang YT, et al. Asymmetric dimethylarginine (ADMA) determines the improvement of hepatic endotelial dysfunction by vitamin $\mathrm{E}$ in cirrhotic rats. Liver Int. 2012;32(1):48-57. https://doi.org/10.1111/j.14783231.2011.02651.x

17. Vairappan B. Endothelial dysfunction in cirrhosis: Role of inflammation and oxidative stress. World J Hepatol. 2015;7(3):443-59. https://doi.org/10.4254/wjh.v7.i3.443

18. Laleman W, Omasta A, Van de Casteele M, et al. A role for asymmetric dimethylarginine in the pathophysiology of por- 
tal hypertension in rats with biliary cirrhosis. Hepatology. 2005;42(6):1382-90. https://doi.org/10.1002/hep.20968

19. Pareja J, Restrepego JC. Métodos diagnósticos en hipertensión portal. Rev Col Gastroenterol. 2016;31:135-45.

20. Kmieć Z. Cooperation of liver cells in health and disease. Adv Anat Embryol Cell Biol. 2001;161:III-XIII, 1-151. https://doi.org/10.1007/978-3-642-56553-3_7

21. Murata S, Maruyama T, Nowatari T, et al. Signal transduction of platelet-induced liver regeneration and decrease of liver fibrosis. Int J Mol Sci. 2014;15(4):5412-25. https:// doi.org/10.3390/ijms15045412

22. Kurokawa T, Zheng YW, Ohkohchi N. Novel functions of platelets in the liver. J Gastroenterol Hepatol. 2016;31(4):745-51. https://doi.org/10.1111/jgh.13244

23. Gangireddy VG, Kanneganti PC, Sridhar S, et al. Management of thrombocytopenia in advanced liver disease. Can J Gastroenterol Hepatol. 2014;28(10):558-64. https://doi.org/10.1155/2014/532191

24. Kaushansky K. Thrombopoiesis. Semin Hematol. 2015;52(1):4-11. https://doi.org/10.1053/j.seminhematol.2014.10.003

25. Hitchcock IS, Kaushansky K. Thrombopoietin from beginning to end. Br J Haematol. 2014;165(2):259-68. https:// doi.org/10.1111/bjh.12772

26. Panasiuk A, Prokopowicz D, ZakJ, et al. Reticulated platelets as a marker of megakaryopoiesis in liver cirrhosis; relation to thrombopoietin and hepatocyte growth factor serum concentration. Hepatogastroenterology. 2004;51(58):1124-8.

27. McHutchison JG, Dusheiko G, Shiffman ML, et al. Eltrombopag for thrombocytopenia in patients with cirrhosis associated with hepatitis C. NEnglJMed.2007;357(22):222736. https://doi.org/10.1056/NEJMoa073255

28. Afdhal NH, Dusheiko GM, Giannini EG, et al. Eltrombopag increases platelet numbers in thrombocytopenic patients with $\mathrm{HCV}$ infection and cirrhosis, allowing for effective antiviral therapy. Gastroenterology. 2014;146(2):442-52.e1. https:// doi.org/10.1053/j.gastro.2013.10.012

29. Afdhal NH, Giannini EG, Tayyab G, et al. Eltrombopag before procedures in patients with cirrhosis and thrombocytopenia. N Engl J Med. 2012;367(8):716-24. https://doi. org/10.1056/NEJMoa1110709

30. Peck-Radosavljevic M. Thrombocytopenia in liver disease. Can J Gastroenterol. 2000;14 Suppl D:60D-6D. https:// doi.org/10.1155/2000/617428

31. Boyer TD, Habib S. Big spleens and hypersplenism: fix it or forget it? Liver Int. 2015;35(5):1492-8. https://doi. org/10.1111/liv.12702

32. Kedia S, Goyal R, Mangla V, et al. Splenectomy in cirrhosis with hypersplenism: improvement in cytopenias, Child's status and institution of specific treatment for hepatitis C with success. Ann Hepatol. 2012;11(6):921-9.

33. Akahoshi T, Tomikawa M, Kawanaka H, et al. Laparoscopic splenectomy with interferón therapy in 100 hepatitis-Cvirus-cirrhotic patients with hypersplenism and thrombocytopenia. J Gastroenterol Hepatol. 2012;27(2):286-90. https://doi.org/10.1111/j.1440-1746.2011.06870.x
34. Nakamura T, Sakata R, Ueno T, et al. Inhibition of transforming growth factor beta prevents progression of liver fibrosis and enhances hepatocyte regeneration in dimethylnitrosamine-treated rats. Hepatology. 2000;32(2):247-55. https://doi.org/10.1053/jhep.2000.9109

35. Nomura Y, Kage M, Ogata T, et al. Influence of splenectomy in patients with liver cirrhosis and hypersplenism. Hepatol Res. 2014;44(10):E100-9. https://doi.org/10.1111/hepr.12234

36. Monroe DM, Hoffman M. The coagulation cascade in cirrhosis. Clin Liver Dis. 2009;13(1):1-9. https://doi. org/10.1016/j.cld.2008.09.014

37. Prelipcean CC, Fierbinteanu-Braticevici C, Drug VL, et al. Liver cirrhosis--procoagulant stasis. Rev Med Chir Soc Med Nat Iasi. 2011;115(3):678-85.

38. Muciño-Bermejo J, Carrillo-Esper $\mathrm{R}$, Uribe $\mathrm{M}$, et al. Coagulation abnormalities in the cirrhotic patient. Ann Hepatol. 2013;12(5):713-24.

39. Donaldson GW, Davies SH, Darg A, et al. Coagulation factors in chronic liver disease. J Clin Pathol. 1969;22(2):199-204. https://doi.org/10.1136/jcp.22.2.199

40. Mariani G, Bernardi F. Factor VII deficiency. Semin Thromb Hemost. 2009;35(4):400-6. https://doi. org/10.1055/s-0029-1225762

41. Zhang C, Wang Q, Zhang T, et al. Liver cirrhosis with factor VII deficiency: a case report and literature review. Zhonghua Gan Zang Bing Za Zhi. 2014;22(1):73-4.

42. De Stefano V, Martinelli I. Splanchnic vein thrombosis: clinical presentation, risk factors and treatment. Intern Emerg Med. 2010;5(6):487-94. https://doi.org/10.1007/s11739010-0413-6

43. Darwish Murad S, Plessier A, Hernandez- Guerra M, Faris F, Eapen CE, Bahr MJ, Trebicka J, et al. Etiology, management and outcome of the Budd-Chiari syndrome. Ann Intern Med. 2009;151(3):167-175.

44. Bianchini M, De Pietri L, Villa E. Coagulopathy in liver diseases: complication or therapy? Dig Dis. 2014;32(5):609-14. https://doi.org/10.1159/000360514

45. Zocco MA, Di Stasio E, De Cristofaro R, et al. Thrombotic risk factors in patients with liver cirrhosis: correlation with MELD scoring system and portal vein thrombosis development. J Hepatol. 2009;51(4):682-9. https://doi. org/10.1016/j.jhep.2009.03.013

46. Valla D. Splanchnic vein thrombosis. Semin Thromb Hemost. 2015;41(5):494-502. https:// doi.org/ 10.1055/ s-0035-1550439

47. Wani ZA, Bhat RA, Bhadoria AS, et al. Extrahepatic portal vein obstruction and portal vein thrombosis in special situations: Need for a new classification. Saudi J Gastroenterol. 2015;21(3):12938. https://doi.org/10.4103/1319-3767.157550

48. Monereo Muñoz M, Aguilera García SG, de la Barreda Heusser R, et al. Cryptogenetic liver cirrhosis and prothrombotic mutations - A mere association? Rev Esp Enferm Dig. 2016;108(9):588-91.

49. Lenting PJ, Christophe OD, Denis CV. von Willebrand factor biosynthesis, secretion, and clearance: connecting 
the far ends. Blood. 2015;125(13):2019-28. https://doi. org/10.1182/blood-2014-06-528406

50. Kujovich JL. Coagulopathy in liver disease: a balancing act. Hematology Am Soc HematolEduc Program. 2015;2015:2439. https://doi.org/10.1182/asheducation-2015.1.243

51. Uemura M, Fujimura Y, Ko S, et al. Pivotal role of ADAMTS13 function in liver diseases. Int J Hematol. 2010;91(1):20-9. https://doi.org/10.1007/s12185-009-0481-4

52. Zheng XL.ADAMTS13 and von Willebrand factor in thrombotic thrombocytopenic purpura. Annu Rev Med. 2015;66:21125. https://doi.org/10.1146/annurev-med-061813-013241

53. Uemura $M$, Matsuyama $T$, Ishikawa $M$, et al. Decreased activity of plasma ADAMTS13 may contribute to the development of liver disturbance and multiorgan failure in patients with alcoholic hepatitis. Alcohol Clin Exp Res. 2005;29(12 Suppl):264S-71S. https://doi.org/10.1097/01. alc.0000192326.08931.cb

54. Matsuyama T, Uemura $M$, Ishikawa $M$, et al. Increased von Willebrand factor over decreased ADAMTS13 activity may contribute to the development of liver disturbance and multiorgan failure in patients with alcoholic hepatitis. Alcohol Clin Exp Res. 2007;31(1 Suppl):S27-35. https:// doi.org/10.1111/j.1530-0277.2006.00283.x

55. D'Alessio S, Genua M, Vetrano S. The protein C pathway in intestinal barrier function: challenging the hemostasis paradigm. Ann N Y Acad Sci. 2012;1258:78-85. https://doi. org/10.1111/j.1749-6632.2012.06557.x

56. Suleiman L, Négrier C, Boukerche H. Protein S: a multifunctional anticoagulant vitamin $\mathrm{K}$-dependent protein at the crossroads of coagulation, inflammation, angiogenesis, and cancer. Crit Rev Oncol Hematol. 2013;88(3):637-54. https://doi.org/10.1016/j.critrevonc.2013.07.004

57. Dahlbäck B. Progress in the understanding of the protein $C$ anticoagulant pathway. Int J Hematol. 2004;79(2):109-16. https://doi.org/10.1532/IJH97.03149

58. Denninger MH, Chait Y, Casadevall N, et al. Cause of portal or hepatic venous thrombosis in adults: the role of multiple concurrent factors. Hepatology. 2000;31(3):587-91. https://doi.org/10.1002/hep.510310307

59. van der Meer JH, van der Poll T, van 't Veer C. TAM receptors, Gas6, and protein S: roles in inflammation and hemostasis. Blood. 2014;123(16):2460-9. https://doi. org/10.1182/blood-2013-09-528752

60. Pobbati AV, Hong W. Emerging roles of TEAD transcription factors and its coactivators in cancers. Cancer Biol Ther. 2013;14(5):390-8. https://doi.org/10.4161/cbt.23788

61. Colomo A, Puente A. Corrección de la coagulación en la hemorragia por varices en el paciente con cirrosis. ¿Es útil? Gastroenterol Hepatol Contin 2011;10:175-9. https://doi. org/10.1016/S1578-1550(11)70039-0

62. Muciño-Bermejo J, Carrillo-Esper R, Uribe $\mathrm{M}$, et al. Coagulation abnormalities in the cirrhotic patient. Ann Hepatol. 2013;12(5):713-24.
63. Vermeer C, Schurgers LJ. A comprehensive review of vita$\min \mathrm{K}$ and vitamin $\mathrm{K}$ antagonists. Hematol Oncol Clin North Am. 2000;14(2):339-53. https://doi.org/10.1016/ S0889-8588(05)70137-4

64. Booth SL, O'Brien-Morse ME, Dallal GE, et al. Response of vitamin $\mathrm{K}$ status to different intakes and sources of phylloquinone-rich foods: comparison of younger and older adults. Am J Clin Nutr. 1999;70(3):368-77.

65. Shearer MJ. Vitamin K deficiency bleeding (VKDB) in early infancy. Blood Rev. 2009;23(2):49-59. https://doi. org/10.1016/j.blre.2008.06.001

66. Shearer MJ, Fu X, Booth SL. Vitamin K nutrition, metabolism, and requirements: current concepts and future research. Adv Nutr. 2012;3(2):182-95. https://doi. org/10.3945/an.111.001800

67. Larson AE, Friedman PA, Suttie JW. Vitamin K-dependent carboxylase. Stoichiometry of carboxylation and vitamin $\mathrm{K}$ 2,3-epoxide formation. J Biol Chem. 1981;256(21):11032-5.

68. von Kries R, Hachmeister A, Göbel U. Can 3 oral 2 mg doses of vitamin $\mathrm{K}$ effectively prevent late vitamin $\mathrm{K}$ deficiency bleeding? Eur J Pediatr. 1999;158 Suppl 3:S183-6. https:// doi.org/10.1007/PL00014352

69. Cornelissen M, von Kries R, Loughnan P, et al. Prevention of vitamin $\mathrm{K}$ deficiency bleeding: efficacy of different multiple oral dose schedules of vitamin K. Eur J Pediatr. 1997;156(2):12630. https://doi.org/10.1007/s004310050570

70. Gonsalves WI, Pruthi RK, Patnaik MM. The new oral anticoagulants in clinical practice. Mayo Clin Proc. 2013;88(5):495511. https://doi.org/10.1016/j.mayocp.2013.03.006

71. Bounameaux $\mathrm{H}$. The novel anticoagulants: entering a new era. Swiss Med Wkly. 2009;139(5-6):60-4.

72. Ageno W, Gallus AS, Wittkowsky A, et al. Oral anticoagulant therapy: antithrombotic therapy and prevention of thrombosis, 9th ed: American College of Chest Physicians Evidence-Based Clinical Practice Guidelines. Chest. 2012;141(2 Suppl):e44S-88S.

73. Wada H, Usui M, Sakuragawa N. Hemostatic abnormalities and liver diseases. Semin Thromb Hemost. 2008;34(8):772-8. https://doi.org/10.1055/s-0029-1145259

74. Lisman T, Leebeek FW. Hemostatic alterations in liver disease: a review on pathophysiology, clinical consequences, and treatment. Dig Surg. 2007;24(4):250-8. https:// doi.org/10.1159/000103655

75. Martí-Carvajal AJ, Solà I. Vitamin K for upper gastrointestinal bleeding in patients with acute or chronic liver diseases. Cochrane Database Syst Rev. 2012;(9):CD004792. https://doi.org/10.1002/14651858.CD004792.pub4

76. Barton CA. Treatment of coagulopathy related to hepatic insufficiency. Crit Care Med. 2016;44(10):1927-33. https://doi.org/10.1097/CCM.0000000000001998

77. Ditisheim S, Goossens N, Spahr L, et al. Coagulation and cirrhosis: new insight. Rev Med Suisse. 2012;8(352):1652, 1654-6. 